\title{
Study on Vibration Damping Mechanism of Shoe Sole with Alternating Lattice Structure Using Vibration Level Difference
}

\author{
Chongning Wang $\mathbb{D},{ }^{1}$ Xiaoying Liu $\left(\mathbb{D},{ }^{1}\right.$ Yong Yue $\mathbb{D},{ }^{1}$ Jiazan Huang $\mathbb{D},{ }^{1}$ Xianwei Huang, ${ }^{2}$ \\ and Bin Liu'
}

${ }^{1}$ College of Mechanical Engineering and Automation, Huaqiao University, Xiamen, China

${ }^{2}$ The First Affiliated Hospital of Xiamen University, Xiamen 361003, China

Correspondence should be addressed to Xiaoying Liu; yinglxy@hqu.edu.cn

Received 26 December 2020; Revised 20 August 2021; Accepted 23 August 2021; Published 20 September 2021

Academic Editor: Chiara Bedon

Copyright (c) 2021 Chongning Wang et al. This is an open access article distributed under the Creative Commons Attribution License, which permits unrestricted use, distribution, and reproduction in any medium, provided the original work is properly cited.

\begin{abstract}
As one of the media of the ground and feet, the design of footwear products has lately received great attention, and the cushioning performance of the sole has become a key factor for the comfort and sportiness of the foot. In this paper, a new type of middle sole sports shoes with an alternating gradient lattice structure was proposed. The dynamic response of the structure was analyzed by ABAQUS software, and the model was validated by modal analysis. The effects of different kinds of alternating lattice and uniform lattice sole models on vibration isolation were analyzed by using the vibration level difference as the evaluation index of vibration characteristics. The analysis results are as follows: (a) We found that the mean of the vibration level difference of the alternating gradient structure is higher than that of the uniform lattice structure, which confirms the feasibility of the alternating gradient arrangement and its excellent buffering performance. (b) Two kinds of vibration stage drop values of the 24-series alternating lattice structure model are analyzed, and "C-G-X" structure has the highest vibration stage drop value. In addition, the comprehensive analysis of the alternating gradient lattice structure of the soles shows that the four types of structures have good cushioning performance, and the $\mathrm{C}$-series structure in the frequency range of $0-140 \mathrm{~Hz}$ vibration level difference value is higher than other series. The results show that the evaluation index of vibration level difference based on mechanical vibration characteristics can accurately analyze the response of different structure soles to vibration, which also provides a method for the future design of vibration reduction and exploration of the biomechanics of footwear.
\end{abstract}

\section{Introduction}

1.1. Structural Vibration. Vibration reduction of athletic shoes is a hot research field in sports biomechanics and ergonomics. The material and structure of the sole are important factors affecting the vibration isolation performance of athletic shoes. In terms of materials, special materials with excellent damping properties are used in the middle soles of shoes, for example, heated compressed EVA sealed microcellular foam materials, high-density $\mathrm{PU}$ foaming materials, PVC materials, or materials made from a combination of these. When the shoe is impacted, the deformation and energy absorption characteristics of this special material achieve the purpose of shock absorption. Lin et al. $[1,2]$ explored the influence of EVA material thickness, hardness, combination mode, and other parameters on the buffer performance of foam materials. Gross and Bunch [3] evaluated the impact load capacity of the midsole of athletic shoes made of three materials through special sensors, and the results showed that there were significant differences in pressure and cushioning capacity in different areas of the sole. In the endurance test of materials, Verdejo et al. [4] used the finite element method to analyze the pressure distribution and fatigue of the insole of running shoes made of EVA foam. The results showed that the peak plantar pressure increased significantly after $500 \mathrm{~km}$, and structural damage appeared in EVA foam after $750 \mathrm{~km}$.

Traditional soles take advantage of the soft properties of the material to achieve cushioning. In fact, structural improvement is more important in reducing the vibration of 
the sole. The midsole is an important component to ensure shock absorption and regression of energy. Sterzing [5] carried out biomechanical tests on EVA comprehensive training shoes with different midsole bubbles and analyzed the vibration reduction and buffer performance through the peak value of vertical ground reaction force. The results showed that the vibration reduction effect was better than the existing EVA sports shoes. In order to enhance the regression of energy performance, Nike launched the "Air Force" and "Zoom-Air" sneakers, in which the airbag structure will greatly improve the resilience of the soft material. New Balance designs a special construction, polyurethane wrapped EVA, for increased rigidity and shock absorption. Adidas has introduced the A3 [6] system, which improves its cushioning capability through a grid-shaped structural module. The "Li Ning Bow" sole introduced by Li Ning [7] adopts the arch structure, which effectively alleviates the ground impact and improves the kinetic energy regression effect. It has become a hot research topic to design and research soles vibration reduction based on structure and material. Most of the existing vibration evaluation indexes are based on the analysis of pressure distribution in the time domain. In fact, compared with the pressure analysis, the vibration level difference can better reflect the amplitude response of different regions, but this kind of vibration isolation index is rarely analyzed in the shoe. In an effort to overcome this challenge, we used an unconventional approach, dynamic analysis of soles vibration mechanism of different structures by vibration level difference.

1.2. Lattice Structure. At present, some scholars have carried out the design and research work of lattice structure midsole. Saucony [8] has designed a special grid structure, inserting a function box in the middle of the sneaker, and the ropes are interwoven around the outer frame to achieve the effect of absorbing the impact like a racket. Adidas has launched the Future Craft 4D $[9,10]$. Using the Clip3D printing technology, it has made the midsole sports shoes with a cellular honeycomb structure, which has the advantages of high elasticity and lightweight. Jones et al. [11] designed an internal structure composed of a number of small blocks of polyurethane material, with complex lever connections between the blocks, which produce different cushioning effects with the change of partial pressure between the foot and the ground, providing sufficient support and elastic deformation. Christensen et al. [12] investigated 2D and 3D low-density lightweight materials and showed that they could produce specific mechanical properties in the form of cellular, lattices, and sandwiches, such as high stiffness, high strength, and negative Poisson's ration performance. Zhang et al. [13-19] studied the buffering performance of the sandwich structure and honeycomb structure. Among them, the lattice structure, because of its large surface area and porosity, plays a lightweight, high strength, impact resistance, and high energy absorption of mechanical properties and is widely used in light design, shock absorption, and other engineering fields. Therefore, this kind of lattice structure provides the design idea for this paper. Evans et al. [20] found that the energy absorption performance of lattice structure is better than that of isotropic multicellular structures. The design and topology optimization of lattice structure by Wang et al. [21] can significantly improve the stiffness and natural frequency. In the intersected field of biomechanics and structure, there are also some researches: Kolken et al. [22, 23], starting from orthopedic implants, proposed a lattice structure with various parameters to obtain better compression performance and achieve elastic modulus similar to that of human bones. Tang et al. [24, 25] established the relationship between sneakers and biomechanics and discussed the dynamic stability of limbs. Compared with the lattice structure with uniform density, the properties of gradient lattice will change with the change of region due to the change of structural porosity [26]. Niknam et al. [27] found that when the strain increases, the stiffness and energy absorption characteristics of the gradient lattice can exceed those of the lattice structure with uniform density distribution. Ajdari et al. [28] introduced density gradient and compared the dynamic properties of lattice structures with different rules to develop a new shockproof structure. Peng et al. [29, 30] introduced functional gradients and explored the relationship between porosity and structural mechanical properties in lattice structures.

At present, most researches only focus on the parameter design or dynamic response analysis of lattice structure, while the literature on the vibration mechanism exploration of the combination of lightweight structure design and biomechanics is very rare. Therefore, based on the above contents, this paper proposes a hypothesis: the lattice structure is combined with the way of alternating gradient, and the lightweight structure is filled into the sneaker midsole. Based on the evaluation index of vibration characteristics of the vibration level drop, the dynamic analysis of the vibration response of the midsoles and heels of shoes was carried out by the finite element method, and innovation was made in the cross-field of biomechanics and structural mechanics to explore the biomechanical effect and shock absorption effect of various alternating lattice structures on human feet.

\section{Design and Methods}

2.1. Structure Design. In this paper, in order to realize the alternating gradient type lattice structure, four typical lattice structures are designed by connecting the nodes, edges, body centers, face centers, and diagonal key points of the cube. As shown in Figure 1(a), "Grid" is a basic lattice structure composed of 12 edges. In Figure 1(b), "Cross" is composed of diagonals of six faces to express the mechanical properties of the faces. In Figure 1(c), "X" is a cross-lattice structure composed of the body center and 8 nodes, which can well transmit lattice loads to the center node. In Figure 1(d), "Star" can be seen as a combination of a "Grid" and an "X" structure that enhances the load capacity at the endpoints of the lattice structure.

2.2. Alternating Structure. In this paper, the same lattice structure is arranged horizontally in a number of $10^{*} 10$. Three different lattice structures are arranged along the $z$-axis and 


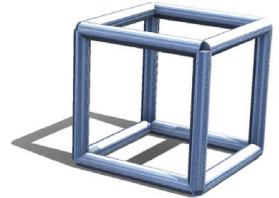

(a)

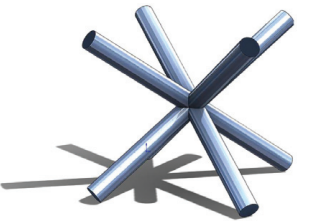

(c)

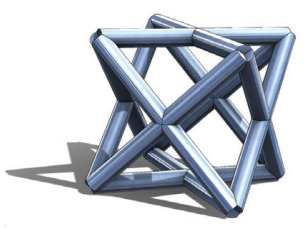

(b)

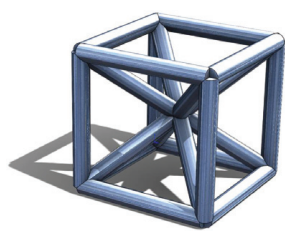

(d)

FIgURE 1: Lattice type.

placed on the middle sole of the shoe. In consideration of the connectivity between different lattices, each lattice is connected with a splint in the $z$-axis direction, and the splint and the lattice are of the same material. Four types of alternating structures, namely, Group C, Group G, Group S, and Group $\mathrm{X}$, can be obtained by naming and grouping the bottom lattice types. Each group of 6 kinds of alternating gradient structure can be obtained, with a total of $A_{4}^{3}=24$ kinds of alternating structure. The thickness and pillar diameter is $1 \mathrm{~mm}$, and the side length of the cube of a lattice unit is $6 \mathrm{~mm}$. As shown in Figure 2, the structure types from bottom to top are Cross, $\mathrm{X}$, and Grid, so it can be named as "C-X-G" structure.

2.3. Vibration Evaluation Index. At present, the commonly used parameters to measure the vibration transmission characteristics [31, 32] are force transmissibility, insertion loss, vibration intensity, power flow, peak counterforce, peak negative acceleration, vibration level difference, and so forth.

Among them, the vibration level difference can be directly obtained through the actual measurement [33]. In particular the lattice structure with multiple support types can reflect the information of vibration isolation efficiency and give the attenuation effect of the structure to the elastic wave. The greater the vibration level difference is, the better the structure buffering effect will be. In this paper, the acceleration vibration level difference is obtained by using this index.

The theoretical derivation of the vibration level difference is as follows.

According to the sole cushioning process, a two-degreeof-freedom undamped elastic system model is established as shown in Figure 3.

The mass is $m$, the elastic stiffness is $k$, the system excitation is $F$, and the vibration response is $x$. The dynamic differential equation of the system is as follows:

$$
\left\{\begin{array}{l}
m_{1} x_{1}(t)+k_{1}\left[x_{1}(t)-x_{2}(t)\right]=F(t), \\
m_{2} x_{2}(t)+\left(k_{1}+k_{2}\right)\left[x_{2}(t)-x_{1}(t)\right]=0 .
\end{array}\right.
$$

Calculate the particular solution of the vibration system:

$$
\left\{\begin{array}{l}
F(t)=|F| e^{j \omega t}, \\
x_{1}(t)=x_{1} e^{j \omega t}, \\
x_{2}(t)=x_{2} e^{j \omega t}
\end{array}\right.
$$

where

$$
\begin{aligned}
& \omega_{1}=\sqrt{\frac{k_{1}}{m_{1}}}, \\
& \omega_{2}=\sqrt{\frac{k_{1}+k_{2}}{m_{2}}},
\end{aligned}
$$

and from (1), (2), and (3),

$$
\left\{\begin{array}{l}
\omega_{1}^{2}\left(X_{1}-X_{2}\right)-\omega^{2} X_{1}=\frac{1}{m_{1}}|F|, \\
\omega_{2}^{2}\left(X_{2}-X_{1}\right)-\omega^{2} X_{2}=0 .
\end{array}\right.
$$

From (1), (2), (3), and (4),

$$
\left\{\begin{array}{l}
X_{1}=\frac{1}{m} \frac{\omega_{2}^{2}-\omega^{2}}{\omega^{4}-\omega^{2}\left(\omega_{1}^{2}+\omega_{2}^{2}\right)}|F|, \\
X_{2}=\frac{1}{m} \frac{\omega_{2}^{2}}{\omega^{4}-\omega^{2}\left(\omega_{1}^{2}+\omega_{2}^{2}\right)}|F| .
\end{array}\right.
$$

The vibration level difference can be obtained by the vibration response value. According to the definition, the vibration level difference of a vibration system is 10 times the commonly used logarithm of the square of the effective value of the vibration response between the elastic upper reference point and the lower reference point [31]. The vibration response can be displacement, velocity, or acceleration. In this paper, the vibration level difference of acceleration is used as the evaluation index of the vibration system.

The acceleration of vibration level difference stage can be expressed as 


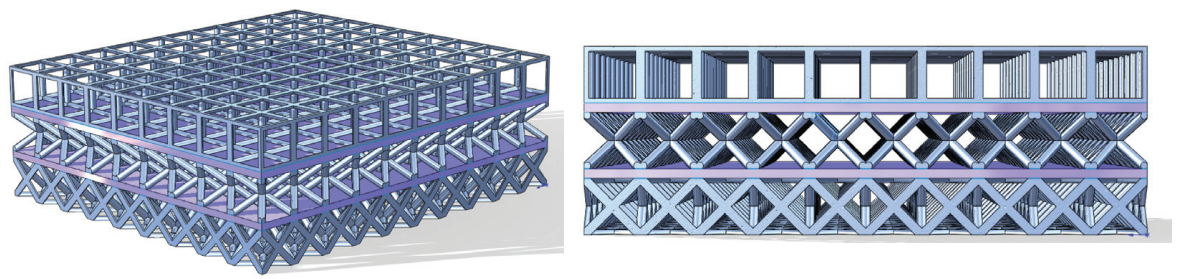

FIGURE 2: Alternating gradient lattice structure.

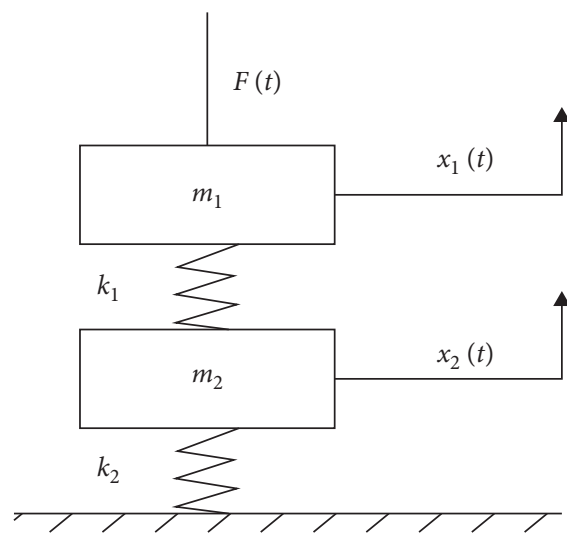

Figure 3: Vibration isolation system model.

$$
L=10 \lg \frac{X_{1}^{2}}{X_{2}^{2}}=20 \lg (D) .
$$

The unit is $d B . D=a_{1} / a_{2}$ is the ratio of vibration level difference of the structure; $a_{1}, a_{2}$ are the maximum displacement accelerations of the upper and lower surface nodes of the sole, respectively.

2.4. Material Models. The sole model in this paper is made of ethylene-vinyl acetate (EVA) copolymer material. Because of its excellent mechanical properties of rigidity, strength, and toughness, as well as excellent machinability, it becomes the best choice of sole material in this paper. After testing, properties of EVA materials are obtained as follows: density $\rho=0.0123 \mathrm{~g} / \mathrm{cm}^{3}$, Young's modulus $E=4 M p a$, and Poisson's ratio $\varepsilon=0.4$.

\section{Finite Element Analysis}

3.1. Validation of the Mesh. Grid density is an important parameter that affects the accuracy and efficiency of calculation. Theoretically speaking, the smaller the mesh size, the denser the nodes and the higher the solution accuracy. However, due to computing resources and rounding errors, the grid cannot be encrypted indefinitely [34].

Therefore, this paper designs sole models with different mesh sizes $(2 \mathrm{~mm}-12 \mathrm{~mm})$ and uses the same boundary conditions as the formal experiment to verify the mesh density. The analysis of convergence results is shown in Figure 4.

As can be seen from Figure 4, with the increase of mesh size, the curves of maximum stress and maximum

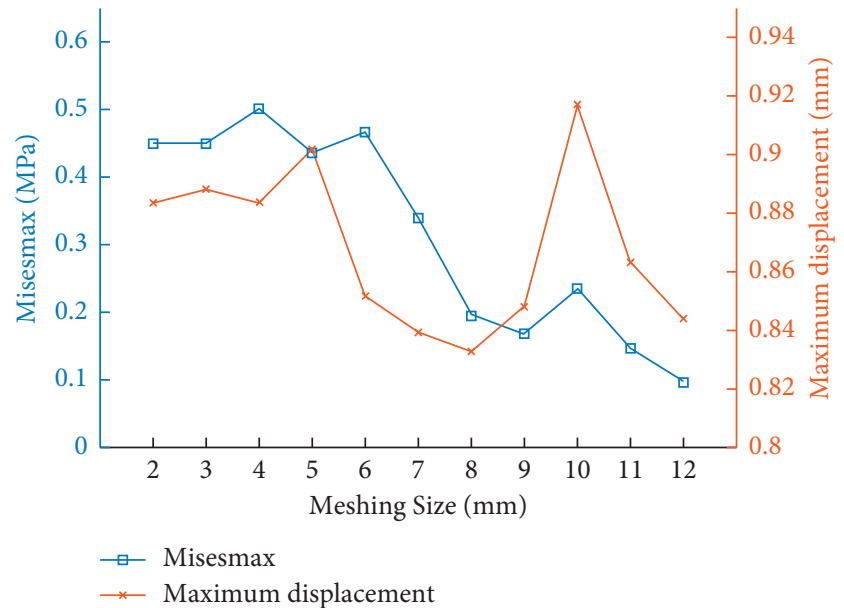

FIgURE 4: Convergence results of soles with different mesh sizes.

displacement of the model fluctuate greatly, especially in the range of $8-12 \mathrm{~mm}$. When the mesh size is less than $5 \mathrm{~mm}$, the fluctuation ratio decreases obviously, and the curve becomes slow and tends to converge. Considering the rationality of calculation cost and the number of grids, this model adopts a $4 \mathrm{~mm}$ grid size, ABAQUS tetrahedral grid structure, and continuous C3D10M element (10-node modified quadratic tetrahedral element), as shown in Figure 5. Finally, the sole model obtained 1186,346 nodes and 670,990 elements, as shown in Figure 6.

3.2. Boundary Conditions of the FEA Model. With the consent of the subject, a CT scan was performed on the right lower limb of a healthy male volunteer (25 years old, $75 \mathrm{~kg}$ ) in the laboratory. The obtained data were imported into the medical graphics processing software Mimics to reconstruct the tibia, talus, and fibular bones, and three-dimensional model reconstruction was performed on multiple bones under the talus (including the radix and wedge-shaped bones). At the same time, ligaments, cartilage, and other models were established and STL files were generated. The STL model was imported into Geomagic Studio for smooth and accurate surface processing (Figure 7) and then transformed into a solid at NX/UG and assembled to get the ideal foot model.

Similarly, 24 models of midsole sneakers and ground models were created using NX/UG. The above model was imported into Abaqus (Figure 8) to set up the interaction between bone, ligament, cartilage, and other tissues. The dynamic response parameters between the foot and the shoe 


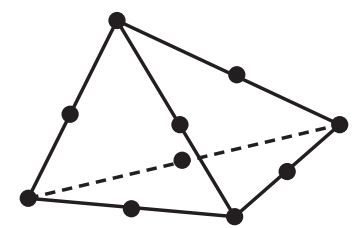

Figure 5: Linear element (C3D10M).

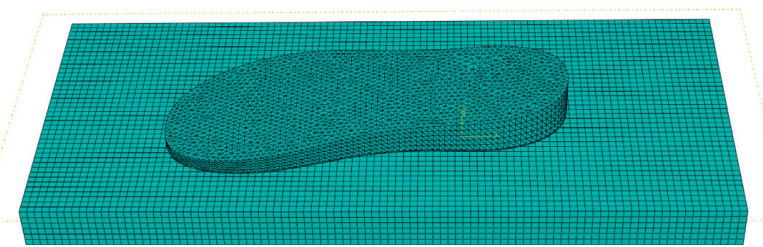

FIgURE 6: Model meshing results.

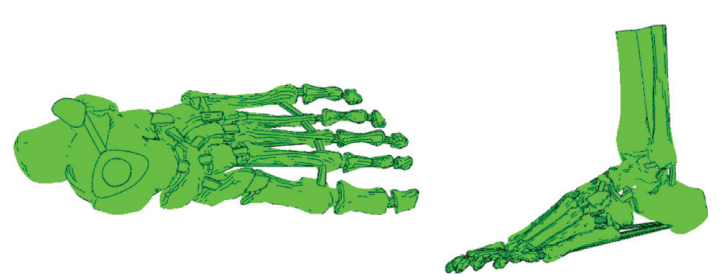

FIgURE 7: Schematic diagram of foot model.

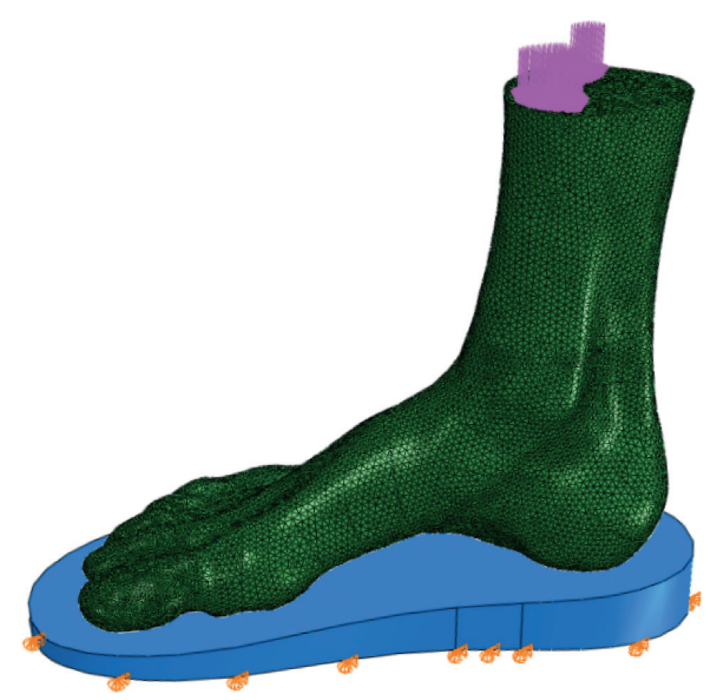

FIgURE 8: A biomechanical finite element model of "foot-shoe.".

surface can be measured by applying the human body gravity (including the pressure generated by bouncing) on the foot model and setting the action time in the analysis step, so as to obtain more accurate experimental data.

This paper adopts an explicit dynamic algorithm to solve transient analysis. In order to reduce the computational time, the model is simplified and only the soles are analyzed because the mesh number of the fem is huge. Boundary conditions were extracted from the original foot-shoe coupling model.
The bottom of the ground is set as a 6-DOF fully fixed constraint. The interlaced lattice structure sole model adopts ideal elastic-plastic material, whose density is $1.23 \mathrm{E}-10 \mathrm{t} /$ $\mathrm{mm} 3$, with elastic modulus of $4 \mathrm{MPa}$ and Poisson's ratio of 0.4 . The ground model has a density of $2.5 \mathrm{E}-9 \mathrm{t} / \mathrm{mm} 3$, an elastic modulus of $17000 \mathrm{MPa}$, and a Poisson's ratio of 0.3 . The contact between shoes and ground is universal, and the friction coefficient is 0.6 according to the empirical formula. The load conditions corresponding to the foot-shoe coupling model are applied to the heel region of the model. A plane is set at a position of $1 \mathrm{~mm}$ vertical to the ground to divide the sole into two parts, and 20 reference points are set, respectively, in the circular area on the upper surface of the two parts for subsequent calculation of vibration level difference. The finite element model is shown in Figure 9.

3.3. Modal Analysis Verification. In this paper, the natural frequencies of different modes are obtained by modal analysis of soles with the finite element method. Through the EDM-Modal analysis software, the force hammer was used for multipoint excitation, and the laser vibrometer was used for the single-point vibration picking-up mode test analysis method, and finally, the natural frequency within the range of $0-400 \mathrm{~Hz}$ of the model was obtained. The real model of EVA sole was put into the suspension device to realize free modal analysis. A number of measuring points are set up in the software to reflect the contour characteristics of the sole model. The modal test site and test points of the analysis model are arranged as shown in Figures 10 and 11.

By comparing the first 10 modes of the finite element model with the experimental model, the relative error of the finite element model calculation was obtained. The results of the experimental mode and the calculated model are shown in Table 1. It can be seen that the modal error between the experimental model and the finite element model of the sole is less than $15 \%$, indicating that the established finite element model of the sole can show its dynamic behavior, and the model can be used for the next finite element analysis.

3.4. Comparison of Experimental and Reference Results. During the compression cushioning of the sole of an alternating gradient lattice structure, the deformation and collapse of the layered lattice structure will show different cushioning capacity and energy absorption characteristics compared with the uniform structure.

In order to verify that the buffer performance of the alternating gradient lattice structure in this paper is better than that of the uniform lattice structure, four groups of reference structures are set up for finite element comparison analysis. The reference group is a three-layer structure with the same lattice arrangement, while the experimental group is the laminated and alternating structure proposed in this paper (Figure 12). For the convenience of analysis, six groups of data in each experimental group were averaged to make a more intuitive comparison of the overall vibration level difference value. 


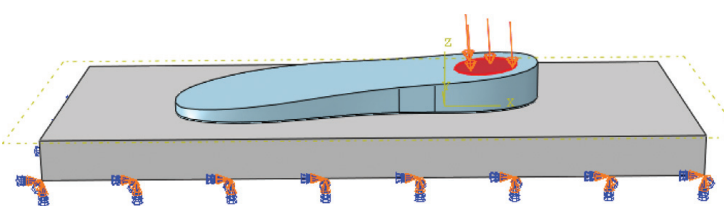

Figure 9: Constraints and loads of finite element models.

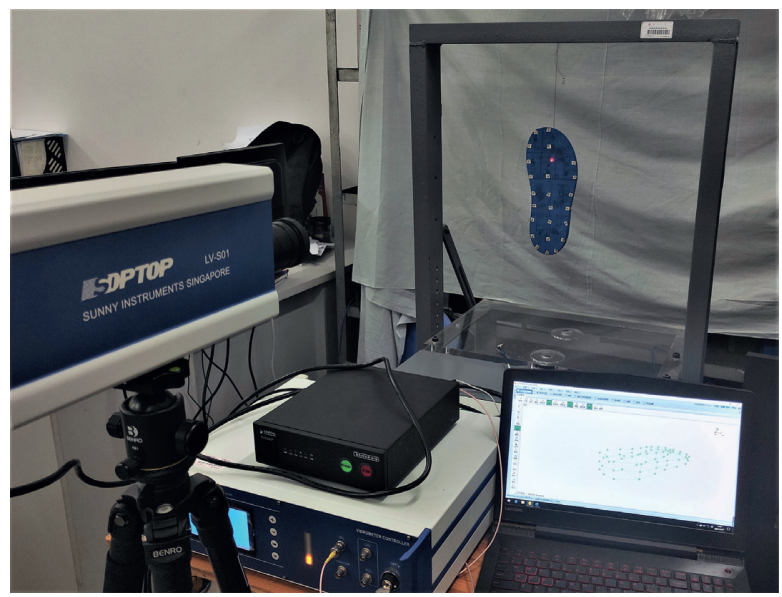

FIGURE 10: The modal experiment platform of the shoe sole.

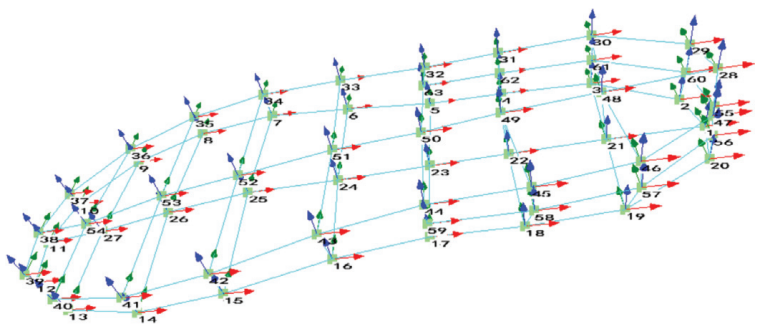

FIGURE 11: Arrangement of measuring points for modal analysis.

TABLE 1: Natural frequencies from experimental and numerical simulation results.

\begin{tabular}{lcccccccccc}
\hline Mode no. & 1 & 2 & 3 & 4 & 5 & 6 & 7 & 8 & 9 & 10 \\
\hline Experimental results $(\mathrm{Hz})$ & 47.6 & 77.9 & 116.6 & 154.9 & 193.1 & 208.1 & 250.3 & 279.7 & 318.2 & 351.9 \\
Numerical results (Hz) & 55.9 & 81.9 & 114.3 & 161.1 & 185.3 & 202.2 & 240.9 & 274.9 & 312.9 & 337.5 \\
$\Delta \mathrm{R} \%$ & -14.85 & -4.88 & 2.01 & -3.85 & 4.21 & 2.92 & 3.90 & 1.75 & 1.69 & 4.27
\end{tabular}

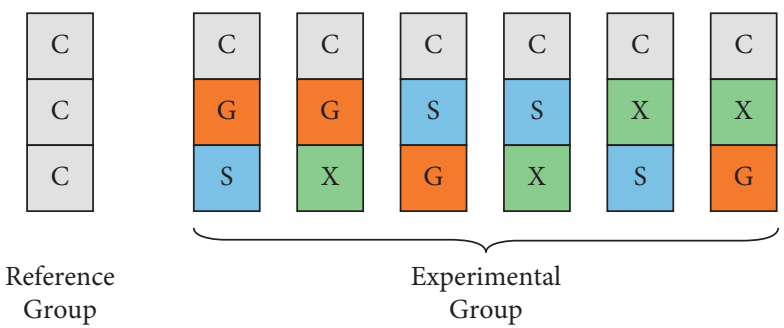

FIgURE 12: Reference and experimental model. 


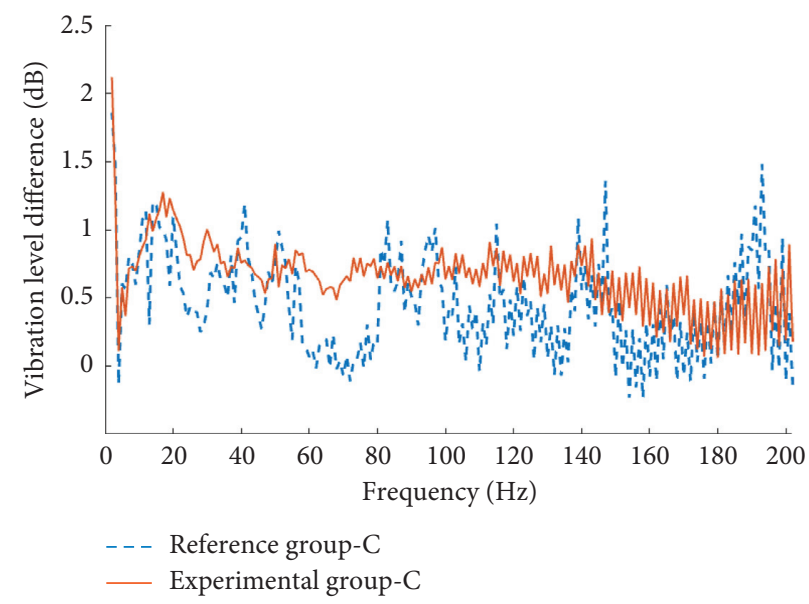

Figure 13: The vibration level difference contrast curve of group C.

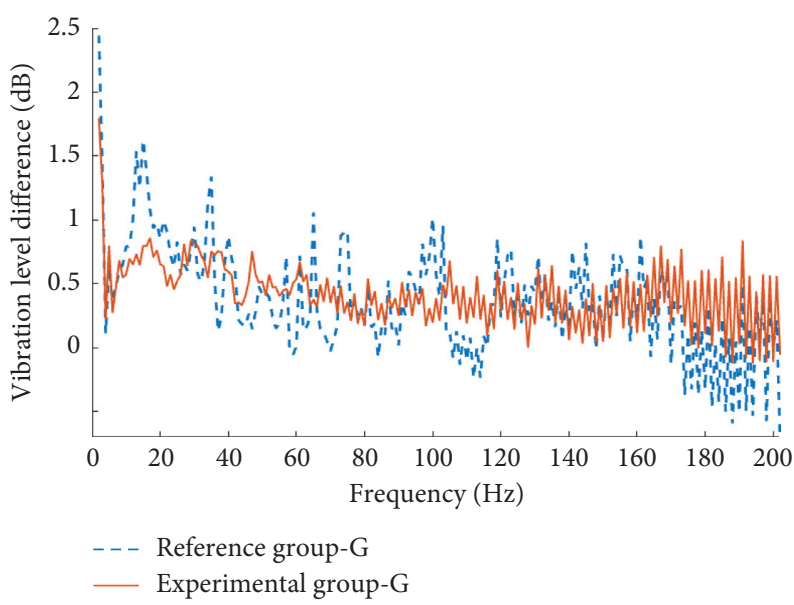

Figure 14: The vibration level difference contrast curve of group G.

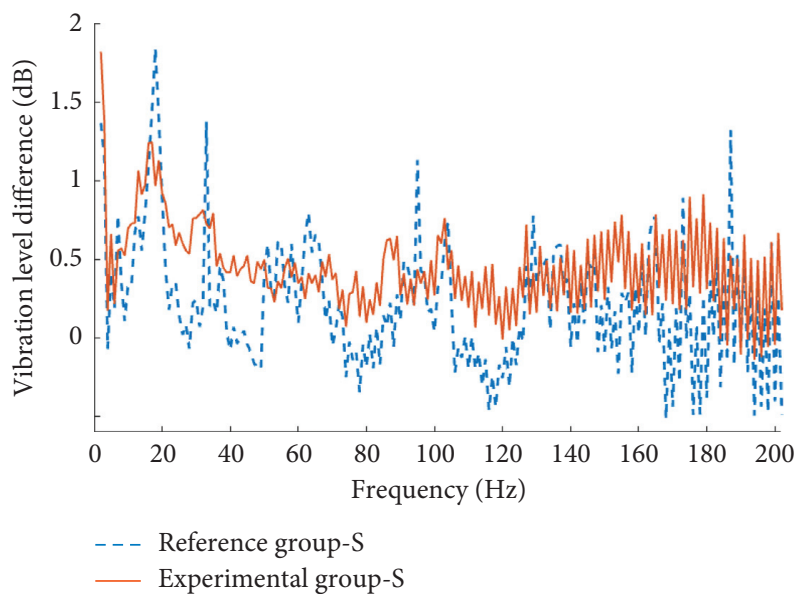

FIgURE 15: The vibration level difference contrast curve of group S.

The average vibration level differences of the experimental groups C, G, S, and X are 0.65, 0.42, 0.45, and 0.47. The average vibration level differences of the reference group C, G, S, and X are $0.45,0.38,0.21$, and 0.34 .
From the data, the damping performance of the alternating structure proposed in this paper is better than that of the uniform structure. From the overall trend of the curve, except for the structure of group G, the value level of the 


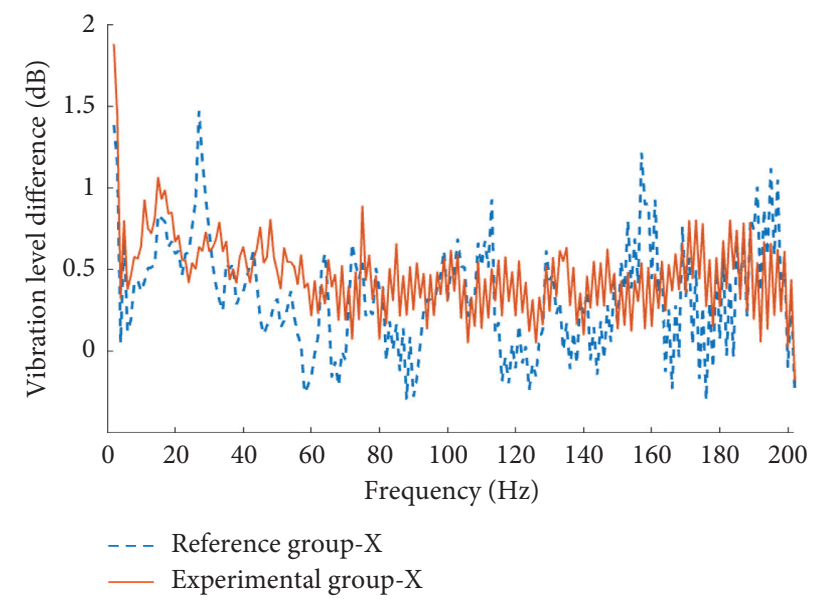

Figure 16: The vibration level difference contrast curve of group X.

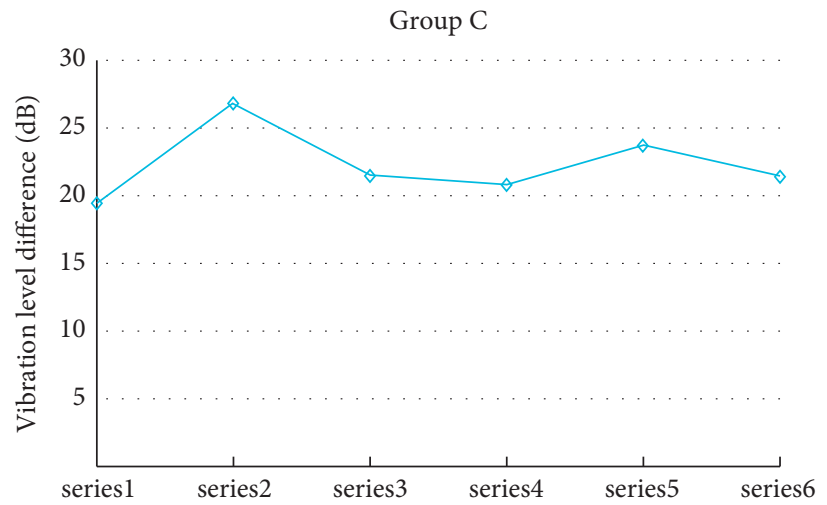

Figure 17: The vibration level difference of group $\mathrm{C}$ with different series.

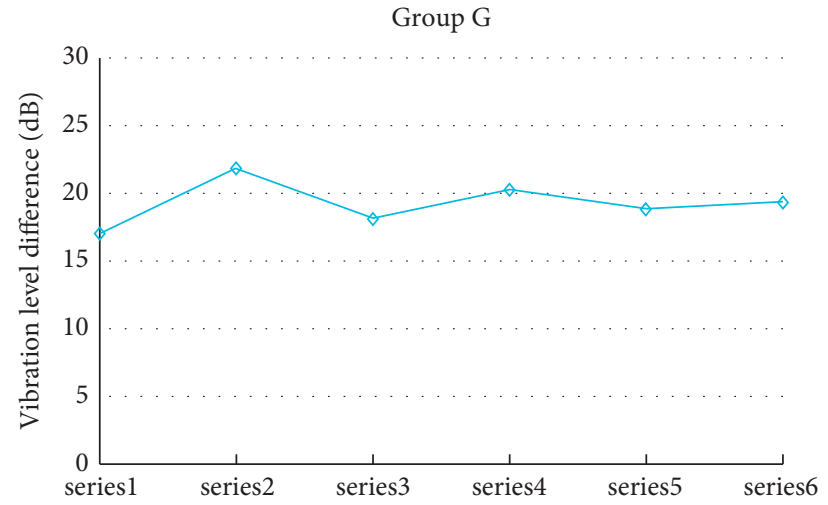

FIGURE 18: The vibration level difference of group $G$ with different series.

reference group is far lower than that of the experimental group. Compared with the single and uniform lattice structure, the gradient lattice design can produce a variety of mechanical properties with regional changes and has good buffering characteristics. The comparative experiment shows the advantages of alternating lattice structure and lays the foundation for the cushioning performance analysis of the following series of gradient lattice structure soles.

\section{Results}

In the course of exercise, the high-intensity vibration brought by shoes can cause different degrees of damage to the sole of the foot. In recent years, studies on foot biomechanics have found that regulating the stress state of the foot bottom is helpful to prevent foot ulcers in patients with diabetic foot, strengthen the protection of soldiers' feet, and 


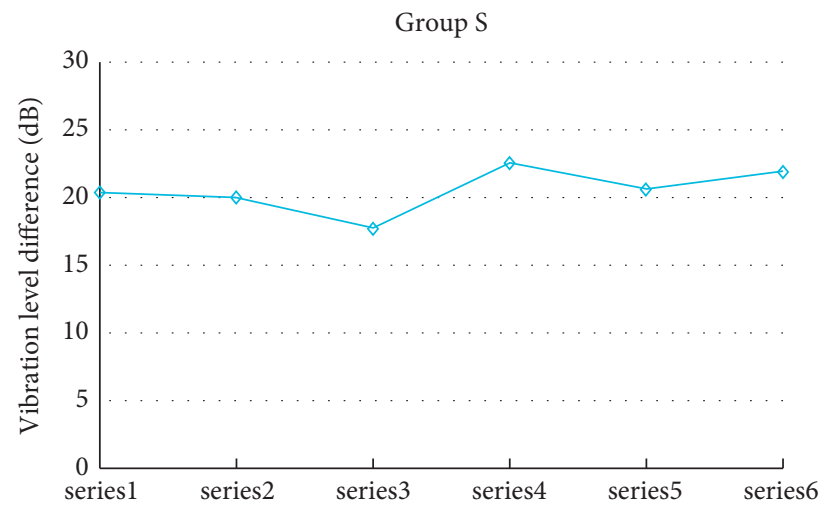

Figure 19: The vibration level difference of group $S$ with different series.

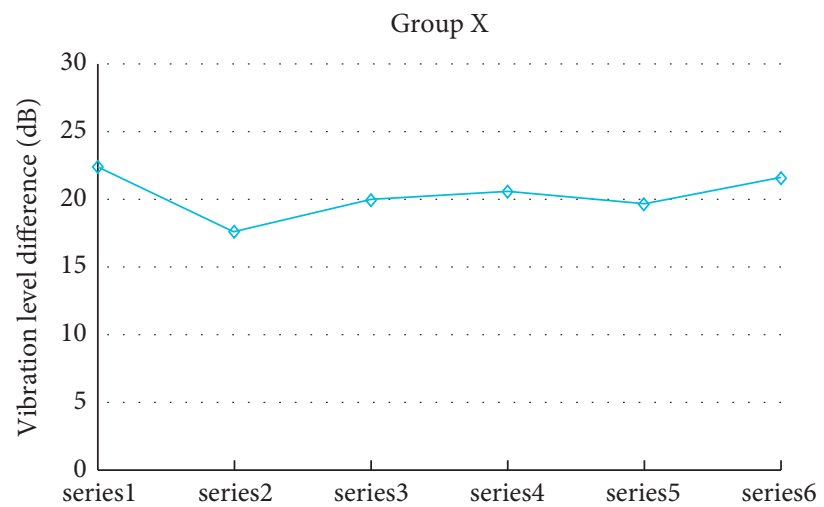

Figure 20: The vibration level difference of group $\mathrm{X}$ with different series.

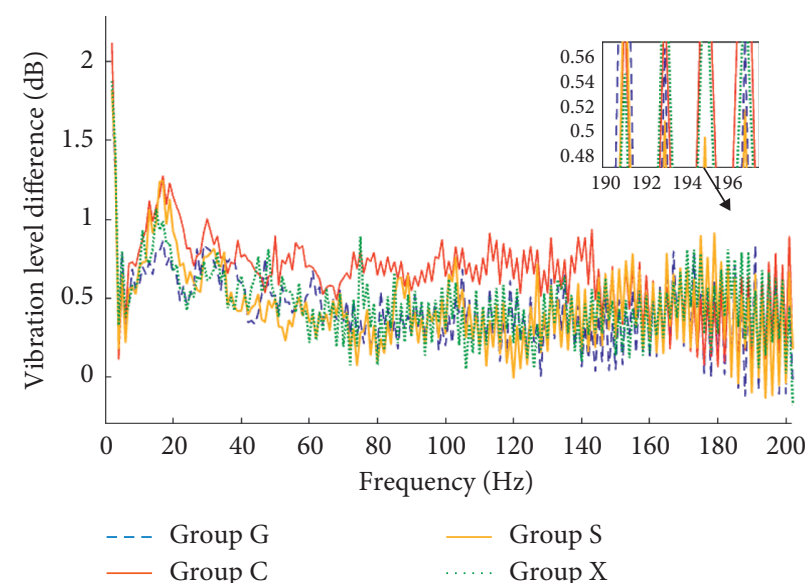

Figure 21: The vibration level difference curves of the four gradient structures.

improve combat ability $[35,36]$. The articles in $[37,38]$ showed that the heel area of the foot has a higher incidence of pressure and foot ulcers and increased the analytical weight of the heel area in the subsequent design of the sole. At the same time, the paper created a porous sole with a single type of lattice, which reduced the peak contact stress by $20 \%$ through structural adjustment. However, most studies still focus on the peak stress or reaction force in the foot. For the characterization of foot and shoe vibration research and analysis, and combined with the fact that multiple types of the alternating lattice structure of lightweight design-related papers are few, in this study, multiple types of the alternating lattice structure of the sole are proposed to reduce the vibration energy transfer in the structure, and the vibration level drop is proposed as the evaluation index of the vibration characteristics of the sole, 
which directly reflects the cushioning performance of the heel area.

4.1. Lattice Structure with "Cross" at the Bottom. Due to the fact that the load direction applied by the model in this paper is vertical to the ground, the vibration isolation effect of the model is mainly reflected in the $z$-axis direction, while the acceleration changes in the $x$-axis and $y$-axis directions are small, which has little influence on the final research results in this paper which can be ignored. Through finite element analysis, the response data of the selected nodes were obtained. According to (6), fast Fourier transform was processed using PyCharm data to convert the results into frequency-domain data, and the broken line diagram of vibration level difference was sorted out by Matlab.

Under this grouping, the overall buffering effect performed well, with an average of $22.25 \mathrm{~dB}$. Among them, the vibration level difference of series 2 "C-G-S" structure reaches $26.79 \mathrm{~dB}$, and it has a better buffer performance. Series 1 "C-G-S" has a low vibration isolation effect.

4.2. Lattice Structure with "Grid" at the Bottom. Compared with group $\mathrm{C}$, the mean drop of the overall vibration level of group $\mathrm{G}$ is $19.27 \mathrm{~dB}$. Among them, the vibration isolation effect of series 2 " $\mathrm{G}-\mathrm{C}-\mathrm{X}$ " is better, reaching $21.85 \mathrm{~dB}$, while series 1 "G-C-S" has a poor buffering effect.

4.3. Lattice Structure with "Star" at the Bottom. The mean value of group $S$ is $20.56 \mathrm{~dB}$, among which the buffering effect of series 4 "S-G-X" is better, reaching $22.46 \mathrm{~dB}$, and series 3 "S-G-C" is slightly insufficient.

4.4. Lattice Structure with " $X$ " at the Bottom. The mean value of group $X$ is $20.30 \mathrm{~dB}$, and the overall trend is stable, in which series 1 "X-C-G" has a better buffering effect and the vibration level difference is $22.39 \mathrm{~dB}$. The vibration isolation capability of series 2 "X-C-S" is slightly inadequate.

In summary, by comparing the four groups of gradient lattice structures, it can be seen that the "C-G-X" structure in the gradient structure has the best buffering effect. In the case that the bottom lattice type is grouped, the buffer performance of group $\mathrm{C}$ is generally higher than those of other groups, while the shock absorption performance of the "G-C-S" structure is the worst among the 24 groups.

4.5. Comparison of Vibration Level Difference between the Four Kinds of Lattice Structures. In this paper, the timedomain data of four groups are transformed into frequencydomain graphs for horizontal analysis, and the average vibration level difference data of six series of each group are selected for overall comparison, which can better reflect the overall trend of buffering performance. At the same time, it also can explore the grouping of the impact of gradient lattice according to the overall data contrast.

It can be seen from the frequency-domain diagram that the curve trends of all groups are similar, and there is no significant difference in the vibration reduction effect of the four groups. Only in the range of $0-140 \mathrm{~Hz}$, the damping buffer ability of group $\mathrm{C}$ is slightly stronger than those of other groups, which is about $0.75 \mathrm{~dB}$. In the $140 \mathrm{~Hz}-200 \mathrm{~Hz}$ range, the buffering performances of the four groups tend to be consistent. In daily footwear application scenarios, vibration with frequencies below $140 \mathrm{~Hz}$ often occurs. At the same time, according to the above results, the vibration stage drops of four types of alternating lattice structures C, G, S, and $\mathrm{X}$ are $44.44 \%, 10.53 \%, 114.29 \%$, and $38.24 \%$ higher than those of uniform structure, respectively. Therefore, based on comprehensive analysis, we believe that the C-G-X structure can provide vibration reduction between shoe systems to a greater extent, and the lattice structure sole design of group $\mathrm{C}$ is more conducive to the mitigation of impact and vibration.

\section{Conclusion}

In this paper, four lattice unit structures and 24 alternating gradient structures are proposed to be applied to the sole heel, and the vibration evaluation index is obtained through acceleration vibration level difference, so as to explore the influence of alternating lattice structure on cushioning performance of sole heel. This paper provides a design idea and vibration analysis method for a vibration-reducing and lightweight sports shoe, which is aimed at reducing the vibration between the foot and the shoe and preventing foot injury.

By setting up a reference group of uniform structures for comparison experiments, it can be reflected that the vibration characteristics of the 24 alternating lattice structures proposed in this paper are stronger than those of the single uniform structure, showing excellent mechanical properties of the alternating structure. According to the vibration level difference value feedback from 20 reference points of the sole heel, among which the vibration isolation effect of series " $\mathrm{C}$ G-X" is much higher than those of other series, it reflects the advantages of the alternating gradient lattice sole design in the aspect of foot cushioning.

The transverse analysis of four groups of alternating lattice structures shows that the vibration damping characteristics of group $\mathrm{C}$ are excellent in the low frequency of $70-140 \mathrm{~Hz}$. In the scenario where footwear is frequently used in daily life, the shock absorption and cushioning capability brought by the "Cross" alternating lattice structure will have a wider application field. Among them, the crystal with alternating gradient arrangement is an innovation in the field of the low-density structure.

This research is a cross innovation in biomechanics and lightweight structural design. Through structural optimization, personalized soles can be prepared to reduce the risk of accidents and bring comfort. The research method of this paper has important theoretical significance and practical value in footwear vibration isolation design, human foot health, and foot and ankle injury prevention. This also provides a method and theoretical basis for the future research direction of foot biomechanics in vibration reduction and buffering. 


\section{Discussion}

The limitations of this study are as follows:

(1) The subject of this study is only a healthy male's foot model, and the original data used for analysis is relatively small. In the future, foot data collection should be carried out for diabetic foot patients, paratroopers, athletes, and other groups prone to a foot injury to improve the reference value of this paper.

(2) The parametric design of alternating gradient lattice structure can be further refined, and the relationship between lattice microtopological parameters and vibration level difference can be established to provide more optimal schemes of shock absorption and buffering.

\section{Data Availability}

No data were used to support this study.

\section{Conflicts of Interest}

The authors declare that they have no conflicts of interest.

\section{Acknowledgments}

This work was supported by the National Natural Science Foundation of China (Grant No. 52175230), Natural Science Foundation of Fujian Province China (Grant No. 2021J01297), Technology Program Pilot Project of Fujian Province (Grant No. 2020H0015), Xiamen Medical and Health Guidance Project (No. 3502720199009), Subsidized Project for Postgraduates' Innovation Fund in Scientific Research of Huaqiao University (Grant No. 18014080034), Research Start-Up Fund Project of Huaqiao University (No. 11BS412), and Subsidized Project for Postgraduates' Innovation Fund in Scientific Research of Huaqiao University (Grant No. 19013080038).

\section{References}

[1] H. Lin, S. Li, W. Tan, and Y. Liu, "Study on the influence factors on cushion resistance of EVA for shoes," Journal of China Leather, vol. 02, p. 019, 2013.

[2] T. L. Grier, J. J. Knapik, D. I. Swedler, and B. H. Jones, "Footwear in the United States Army Band: injury incidence and risk factors associated with foot pain," Journal of The Foot, vol. 12, p. 002, 2010.

[3] T. S. Gross and R. P. Bunch, "Material moderation of plantar impact stress," Medicine \& Science in Sports \& Exercise, vol. 21, no. 5, pp. 619-624, 1989.

[4] R. Verdejo and N. J. Mills, "Heel-shoe interactions and the durability of EVA foam running-shoe midsoles," Journal of Biomechanics, vol. 12, pp. 1379-1386, 2003.

[5] T. Sterzing, K. Thomsen, R. Ding, and J. T.-M. Cheung, "Running shoe crash-pad design alters shoe touchdown angles and ankle stability parameters during heel-toe running," Footwear Science, vol. 7, no. 2, pp. 81-93, 2015.
[6] J. Li, Y. Gu, Y. Lu, and Y. Wang, "Biomechanical research on sports shoe' s core technology," Journal of China Sport Science, vol. 05, pp. 42-51, 2009.

[7] Y. Tang, "Analysis of the design and influence of modern sneaker "device" sole," Journal of HuNan University of technology (Social Science Edition), vol. 02, pp. 4-6, 2008.

[8] H. A. Kim, "Metabolic and performance responses of male runners wearing 3 types of footwear: Nike Vaporfly 4\%, Saucony Endorphin racing flats, and their own shoes," Journal of Sport and Healthence, vol. 11, p. 012, 2020.

[9] Y. Li and Y. Liu, "Development trend of sports shoes design under 3D printing technology," Journal of Packaging Engineering, vol. 24, pp. 152-157, 2018.

[10] J. R. Tumbleston, D. Shirvanyants, N. Ermoshkin et al., "Continuous liquid interface production of 3D objects," Science, vol. 347, no. 6228, pp. 1349-1352, 2015.

[11] C. C. Wang, C. H. Yang, and C. S. Wang, "Feature recognition and shape design in sneakers," Journal of Computers \& Industrial Engineering, vol. 102, pp. 1163-1173, 2016.

[12] R. Christensen, "Mechanics of cellular and other low-density materials," Journal of International Journal of Solids \& Structures, vol. 37, 2000.

[13] D. Wowk, T. Reyno, R. Yeung, and C. Marsden, "An experimental and numerical investigation of core damage size in honeycomb sandwich panels subject to low-velocity impact," Composite Structures, vol. 254, Article ID 112739, 2020.

[14] F. Zhang, J. Song, H. Ma, X. Liu, and Y. Wei, "Effect of interlayer thickness on the model fracture energy release rate of sandwich structure," Journal of Acta Mechanica Sinica.vol. 52, pp. 1080-1094, 2020.

[15] A. H. N. G. Waldley, B. N. A. Fleck, and C. A. G. Evans, "Fabrication and structural performance of periodic cellular metal sandwich structures," Journal of Composites ence \& Technology.vol. 63, no. 16, pp. 2331-2343, 2003.

[16] B. O. Baba, "Curved sandwich composites with layer-wise graded cores under impact loads," Composite Structures, vol. 159, pp. 1-11, 2017.

[17] D. Zhang, Q. Fei, and P. Zhang, "In-plane dynamic crushing behavior and energy absorption of honeycombs with a novel type of multi-cells," Thin-Walled Structures, vol. 117, pp. 199-210, 2017.

[18] H. Niknam and A. H. Akbarzadeh, "In-plane and out-ofplane buckling of architected cellular plates: numerical and experimental study," Composite Structures, vol. 206, pp. 739-749, 2018.

[19] F. Scarpa and Liu, "In-plane elasticity of a novel auxetic honeycomb design," JEC Composites Part B Engineering, vol. 11, 011 pages, 2016.

[20] A. G. Evans, M. Y. He, V. S. Deshpande, J. W. Hutchinson, A. J. Jacobsen, and W. B. Carter, "Concepts for enhanced energy absorption using hollow micro-lattices," International Journal of Impact Engineering, vol. 37, no. 9, pp. 947-959, 2010.

[21] C. Wang, J. Zhu, and M. Wu, "Multi-scale design and optimization for solid-lattice hybrid structures and their application to aerospace vehicle components," Journal of Chinese Journal of Aeronautics, vol. 34, pp. 08-15, 2020.

[22] H. M. A. Kolken, K. Lietaert, T. van der Sloten et al., "Mechanical performance of auxetic meta-biomaterials," Journal of the Mechanical Behavior of Biomedical Materials, vol. 104, Article ID 103658, 2020.

[23] B. J. Ge, C. J. Huang, and B. Y. Lei, "Microstructural features and compressive properties of SLM Ti6Al4V lattice 
structures," Journal of Surface and Coatings Technology, vol. 403, 2020.

[24] Y. Tang, Z. Wang, Y. Zhang et al., "Effect of football shoe collar type on ankle biomechanics and dynamic stability during anterior and lateral single-leg jump landings," Applied Sciences, vol. 10, no. 10, p. 3362, 2020.

[25] N. Sudarmadji, J. Y. Tan, C. K. ChuaLeong, and Y. T. Loh, "Investigation of the mechanical properties and porosity relationships in selective laser-sintered polyhedral for functionally graded scaffolds," Acta Biomaterialia, vol. 7, no. 2, pp. 530-537, 2011.

[26] L. Bai, C. Gong, X. Chen et al., "Mechanical properties and energy absorption capabilities of functionally graded lattice structures: experiments and simulations," International Journal of Mechanical Sciences, vol. 182, Article ID 105735, 2020.

[27] A. H. Niknam and B. A. H. A. Akbarzadeh, "Graded lattice structures: simultaneous enhancement in stiffness and energy absorption," Journal of Materials \& Design, vol. 196, 2020.

[28] A. Ajdari, H. Nayeb-Hashemi, and A. Vaziri, "Dynamic crushing and energy absorption of regular, irregular and functionally graded cellular structures," International Journal of Solids and Structures, vol. 48, no. 3-4, pp. 506-516, 2011.

[29] C. Peng and J. P. Tran, "Bioinspired functionally graded gyroid sandwich panel subjected to impulsive loadings," JEC Composites Part B Engineering, vol. 188, 2020.

[30] R. Alonzo, C. Teo, J. W. Pan, P. S. P. Teng, T. Sterzing, and P. W. Kong, "Effects of basketball shoe midsole hardness on lower extremity biomechanics and perception during drop jumping from different heights," Applied Sciences, vol. 10, no. 10, p. 3594, 2020.

[31] Y. Li, Z. Weng, and J. Tang, "Study on vibration isolation effect evaluation of double layer vibration isolation system on flexible Foundation," Journal of Noise and vibration control, vol. 38, pp. 178-183, 2018.

[32] S. Xu and H. Gui, "Evaluation index of power flow for isolation performance of floating raft system," Journal of Ship Mechanics, vol. 16, pp. 567-572, 2012.

[33] R. Huo and Y. Shi, "Evaluation index of vibration isolation efficiency of power flow and its relation with vibration stage drop," Journal of Shipbuilding of China, vol. 03, pp. 86-92, 2007.

[34] X. Liu, Y. Yue, and X. Wu, "A study of vibration behavior in shoe sole using vibration level difference and power flow method," Journal of the Brazilian Society of Mechanical ences and Engineering, vol. 42, no. 11, pp. 1-17, 2020.

[35] M. Nouman, T. Dissaneewate, and W. Leelasamran, "The insole materials influence the plantar pressure distributions in diabetic foot with neuropathy during different walking activities," Gait \& Posture, vol. 74, no. 5, 2019.

[36] J. D. Simpson, H. Debusk, and C. Hill, "The role of military footwear and workload on ground reaction forces during a simulated lateral ankle sprain mechanism," Foot, vol. 34, p. 53, 2017.

[37] Z. Ma, J. Lin, and X. Xu, "Design and 3D printing of adjustable modulus porous structures for customized diabetic foot insoles," International Journal of Lightweight Materials \& Manufacture, vol. 2, 2018.

[38] L. Tang, L. Wang, and W. Bao, "Functional gradient structural design of customized diabetic insoles," Journal of the Mechanical Behavior of Biomedical Materials, vol. 94, 2019. 